\title{
The lysine methyltransferase Ehmt2/G9a is dispensable for skeletal muscle development and regeneration
}

\author{
Regan-Heng Zhang, Robert N. Judson, David Y. Liu, Jürgen Kast and Fabio M. V. Rossi
}

\begin{abstract}
Background: Euchromatic histone-lysine N-methyltransferase 2 (G9a/Ehmt2) is the main enzyme responsible for the apposition of H3K9 di-methylation on histones. Due to its dual role as an epigenetic regulator and in the regulation of non-histone proteins through direct methylation, G9a has been implicated in a number of biological processes relevant to cell fate control. Recent reports employing in vitro cell lines indicate that Ehmt2 methylates MyoD to repress its transcriptional activity and therefore its ability to induce differentiation of activated myogenic cells.

Methods: To further investigate the importance of G9a in modulating myogenic regeneration in vivo, we crossed Ehmt $2^{\text {floxed }}$ mice to animals expressing Cre recombinase from the Myod locus, resulting in efficient knockout in the entire skeletal muscle lineage (Ehmt $2^{4 \mathrm{myoD}}$ ).

Results: Surprisingly, despite a dramatic drop in the global levels of H3K9me2, knockout animals did not show any developmental phenotype in muscle size and appearance. Consistent with this finding, purified Ehmt $2^{\Delta \mathrm{myoD}}$ satellite cells had rates of activation and proliferation similar to wild-type controls. When induced to differentiate in vitro, Ehmt2 knockout cells differentiated with kinetics similar to those of control cells and demonstrated normal capacity to form myotubes. After acute muscle injury, knockout mice regenerated as efficiently as wildtype. To exclude possible compensatory mechanisms elicited by the loss of G9a during development, we restricted the knockout within adult satellite cells by crossing Ehmt2 ${ }^{\text {floxed }}$ mice to Pax $7^{\text {CreERT2 }}$ and also found normal muscle regeneration capacity.
\end{abstract}

Conclusions: Thus, Ehmt2 and H3K9me2 do not play significant roles in skeletal muscle development and regeneration in vivo.

Keywords: Euchromatic methyltransferase, Ehmt2, Ehmt1, G9a, GLP, Myogenesis, Skeletal muscle, Development, Regeneration, Myod

\section{Background}

The formation of skeletal muscle begins in the embryonic somites [1], which generate the primary myotome and the first primitive myogenic structure containing muscle progenitors. Morphogen gradients including sonic hedgehog (Shh) $[2,3]$ and Wingless (Wnt) [4] ensure initial myogenic specification by controlling the expression of myogenic regulatory factors (MRFs-Myf5, Myod, Myog, and Mrf4) - a conserved family of musclespecific basic helix-loop-helix (bHLH) transcription factors responsible for myogenic lineage commitment and

* Correspondence: fabio@brc.ubc.ca

The Biomedical Research Centre, The University of British Columbia, Vancouver, Canada

(C) 2016 Zhang et al. Open Access This article is distributed under the terms of the Creative Commons Attribution 4.0 International License (http://creativecommons.org/licenses/by/4.0/), which permits unrestricted use, distribution, and reproduction in any medium, provided you give appropriate credit to the original author(s) and the source, provide a link to the Creative Commons license, and indicate if changes were made. The Creative Commons Public Domain Dedication waiver (http://creativecommons.org/publicdomain/zero/1.0/) applies to the data made available in this article, unless otherwise stated. 
MRFs and differentiating to form new myofibers. In mice, this process leads to near-complete tissue regeneration and restoration of muscle function.

Despite much knowledge about the key transcription factors regulating myogenesis, the epigenetic landscapes required for the control of gene expression in skeletal muscle differentiation remain less well understood. Epigenetic mechanisms including different modifications on the unstructured C-terminals (tails) of histone proteins have an increasingly appreciated role in controlling gene expression during myogenesis [5]. Some studies [6-8] have found that specific histone acetyltransferases can interact with Myod and acetylate histones associated with muscle-specific genes, thereby activating their transcription. Recent genome-wide analyses have uncovered dynamic epigenetic changes during myogenesis [9], including the loss of histone 2B (H2B) ubiquitination [10]. Chromatin immunoprecipitation (ChIP)-seq analyses have also revealed the importance of bivalent domains containing both H3K4me1 and H3K27ac in regulating muscle enhancers during myogenesis [9]. These data also point to Myod as playing a key role in the recruitment of chromatin-modifying enzymes and transcription factors to activate such enhancers $[11,12]$.

A less well-characterized histone mark in myogenesis is H3K9me2, produced by euchromatic histone-lysine $N$ methyltransferase 2 (Ehmt2) (MGI: 2148922), also known as G9a. This Su (var)3-9 and enhancer of zeste (SET) domain containing methyltransferase dimerizes with its close homologue Ehmt1 (aka Glp) to induce H3K9me2 [13]. Knockout of Ehmt2 leads to a global reduction of H3K9me2 levels and early embryonic lethality in mice, underscoring its importance and the fact that Ehmt1 cannot fully compensate for its loss [14]. Conditional knockouts of Ehmt2 also demonstrated its importance in germ cell development [15], heart development [16], lymphocyte development [17, 18], leukemia [19], drug addiction [20], cognition, and adaptive behaviors [21, 22]. H3K9me2 appears to occur on repressed genes in euchromatin, whereas H3K9me3 is also a repressive mark, but associated with pericentromeric heterochromatin [23]. The repressive function is led by recruitment of proteins such as HP1, which bind preferentially to H3K9me2 and establish a repressive chromatin conformation [15]. The SET domain in Ehmt2 and other histone methyltransferases also have the potential to methylate non-histone polypeptides [24, 25], allowing possible regulation of the localization and activity of a variety on non-histone proteins [26]. Ehmt2 contains an ankyrin domain, enabling protein-protein interactions [27] with a variety of partners including DNA methyltransferases (DNMT) [28], which provide an alternative mechanism for gene repression [29]. Finally, Ehmt2 can act as a gene activator in addition to its repressive roles, by interacting with coactivators such as in nuclear receptor-mediated transcription regulation $[23,30]$.

Despite Ehmt2 being capable of regulating a diverse range of cellular and biological functions, little is known about its role in skeletal muscle. Working with the widely used murine cell line $\mathrm{C} 2 \mathrm{C} 12$, Ling et al. [31] recently highlighted Ehmt2 as a possible regulator of myogenic differentiation. Using in vitro overexpression and knockdown strategies, Ehmt2 was shown to act as an inhibitor of myotube formation. Biochemical analyses suggested that EHMT2 also has the capability to directly methylate MYOD at K104 [31], revealing a novel Bhlhe41/Sharp1-dependent mechanism inhibiting myogenesis that is controlled by Ehmt 2 through both direct modulation of MYOD and the repressive H3K9me2 modification of Myod target genes [32, 33]. In spite of these initial findings, whether Ehmt2 plays an equivalent role in a more complex biological system such as in vivo skeletal muscle development or regeneration is yet to be evaluated. In this study, we generated transgenic mouse strains to genetically delete Ehmt2 during muscle development as well as in adult satellite cells. We found that proliferation and differentiation of satellite cells was not influenced by the absence of Ehmt2. Knocking out Ehmt2 also failed to result in significant consequences for skeletal muscle development and in adult muscle regeneration in vivo. Thus, Ehmt2 is completely dispensable for the normal functioning, maintenance, and damage response of murine skeletal muscle.

\section{Methods}

\section{Mice and animal care}

C57BL/6 mice harboring the Ehmt $2^{\text {floxed }}$ allele were previously generated by Lehnertz et al. [17]. In this strain, Cre-mediated targeting of the Ehmt $2^{\text {floxed }}$ allele results in a genomic deletion from exon 4 to exon 20 and a frameshift mutation that places the downstream coding sequence out of frame. All other transgenic strains used herein were generated by other groups [34-36] and obtained from The Jackson Laboratory. Inducible Cre recombinase was activated by intraperitoneal injection of tamoxifen dissolved in corn oil $(250 \mathrm{mg} / \mathrm{kg}$ of body weight per day) for 5 consecutive days, followed by 7 days without any treatment to allow sufficient activation. The mice were housed in a pathogen-free facility, and all experiments were performed according to the Canadian Council on Animal Care (CCAC) regulations.

\section{Acute muscle injury}

The tibialis anterior (TA) muscle of 8-12-week-old control or experimental mice was injected with the myotoxin notexin $(7 \mu \mathrm{l})$, as previously described [37]. 


\section{Flow cytometry/FACS}

Skeletal muscle tissue was prepared as described previously [37]. Cell preparations were then incubated with primary antibodies for $30 \mathrm{~min}$ at $4{ }^{\circ} \mathrm{C}$ in supplemented PBS containing $2 \mathrm{mM}$ EDTA and $2 \%$ fetal bovine serum at $\sim 3 \times 10$ [7] cells per milliliter. We used the following monoclonal-conjugated primary antibodies: anti-platelet endothelial cell adhesion molecule (PECAM)-1 (CD31) (clones MEC13.3, Becton Dickinson, and 390, Cedarlane Laboratories); anti-protein tyrosine phosphatase receptor type C (PTPRC) (CD45) (clone 30-F11, Becton Dickinson); anti-lymphocyte antigen 6A/E (LY6A/E) (Sca-1) (clone D7, eBioscience); anti-vascular cell adhesion molecule (VCAM) (produced in-house); and anti-integrin alpha-7 (produced in-house). Satellite cells were identified as PECAM-, PTPRC-, LY6A/E-, VCAM+, and integrin alpha-7+. Antibody dilution and staining volume were determined experimentally. Where necessary, biotinylated primary antibodies were detected using streptavidin coupled to phycoerythrin (PE), allophycocyanin (APC), phycoerythrincyanine 7 tandem complex (PE-Cy7), or fluorescein isothiocyanate (FITC) (Caltag). To assess viability, cells were stained with propidium iodide $(1 \mu \mathrm{g} / \mathrm{ml})$ and Hoechst $33342(2.5 \mu \mathrm{g} / \mathrm{ml})$ and resuspended at $\sim 1 \times 10$ cells $/ \mathrm{ml}$ [7] immediately before flow cytometry analysis or sorting.

Analysis was performed on LSRII (Becton Dickinson) equipped with three lasers. Data were collected using FACSDiva software. Sorts were performed on a FACS Influx (Becton Dickinson) or FACSAria (Becton Dickinson), both equipped with three lasers, using a $100-\mu \mathrm{m}$ nozzle at 18 psi to minimize the effects of pressure on the cells. Sorting gates were strictly defined based on "fluorescence minus one" stains.

\section{Cell culture and immunocytochemistry}

Viable single myofibers were isolated from the extensor digitorum longus (EDL) muscle of 6-8-week-old mice following dissociation with collagenase I as previously described [38]. Myofibers and their associated satellite cells were maintained ex vivo for up to $72 \mathrm{~h}$ in highglucose Dulbecco's modified Eagle's medium (DMEM) supplemented with $20 \% v / v$ FBS, $0.5 \% v / v$ chick embryo extract, and pen-strep. Following culture, single myofibers were fixed in $4 \%$ PFA and then stained overnight with the following primary antibodies: mouse antiPax7 (Developmental Studies Hybridoma Bank (DSHB)), mouse anti-MyoD (Dako, clone 5.8A), mouse antiMyogenin (DSHB, clone FD5).

Fluorescence-activated cell sorting (FACS)-sorted cells were grown in high-glucose DMEM, supplemented with $2.5 \mathrm{ng} / \mathrm{ml} \mathrm{bFGF} \mathrm{(Invitrogen)} 20 \% v / v$ FBS, and $10 \% v / v$ horse serum. This medium is hereafter referred to as "growth medium." Cells were seeded in tissue culturetreated plastics coated with Matrigel (BD Biosciences).
The media were changed every $24-48 \mathrm{~h}$. To induce myogenic differentiation, confluent myoblasts were cultured in DMEM supplemented with $5 \%$ horse serum for up to $96 \mathrm{~h}$, before being fixed in $4 \%$ PFA and stained overnight with mouse anti-Myosin (DSHB, clone MF20).

\section{Histology}

TA muscles were dissected from mice, fixed in $4 \%$ paraformaldehyde overnight followed by $70 \%$ ethanol overnight, and then embedded in paraffin following standard protocols. Tissues were cut with a microtome in a crosssectional orientation through the entire length of the muscle. Cross sections of $5 \mathrm{~mm}$ thickness were then mounted onto glass slides (Thermo Fisher Scientific, USA) and stained with Masson's trichrome or Picrosirius red following standard protocols. The cross-sectional area was used as a measure of myofiber size, which was produced by semi-automated measurements on stitched whole-section images (Nikon).

\section{Gene deletion efficiency measured by allele count}

At least 10,000 FACS-purified satellite cells per sample were used for measuring the efficiency of Ehmt2 conditional knockouts. Cells were lysed and purified for genomic DNA, of which $50 \mathrm{ng}$ per sample was mixed with digital droplet PCR supermix (Bio-Rad) and two TaqMan Copy Number Assays for a duplex (fluorescein amidite (FAM) and VIC) digital droplet PCR assay. The "functional assay" is a TaqMan Copy Number Assay with FAM dye (Thermo Fisher Scientific \#4400291 Mm00466045_cn) that detects Ehmt2 in a region from intron 14 to exon 15, which is found only in the wildtype or floxed alleles (functional alleles) of the gene. The "reference assay" is a TaqMan Copy Number Assay with VIC dye (Thermo Fisher Scientific \#4400291 Mm00466690_cn) that detects Ehmt2 in a region from intron 25 to intron 26, which is found in any null, wildtype, or floxed alleles of the gene. Droplets of the mixture were generated according to standard digital droplet PCR protocol (Bio-Rad) and ran in a thermocycler for 40 PCR cycles. PCR products were read droplet-wise in duplex (FAM and VIC) following standard protocol (BioRad), and a signal ratio was calculated by dividing the absolute copy number of the functional assay to the copy number of the reference assay. The signal ratio was then used to interpolate the functional allele frequency (\%) from a known standard curve (see Additional file 1: Figure S1). The standard curve of signal ratios was produced by performing digital droplet polymerase chain reaction (ddPCR) using genomic DNA that were mixed at known proportions from Ehmt $2^{\text {wt/wt }}$ and Ehmt $2^{\text {null/null }}$ mouse embryonic fibroblasts. The standard curve has a Pearson coefficient of 0.993 , and a statistical test of linearity yielded a $p$ value of 0.0001 . The results of this measurement are presented either as the frequency of functional alleles in each 
experimental sample or as the efficiency of gene deletion, which is calculated as the reciprocal of the functional allele frequency.

\section{Statistics}

Mouse weight measurements plotted against age were subjected to linear regression analysis. A sum-of-squares $F$ test was performed on a shared model to test the null hypothesis that one curve fits all groups. The resulting $p$ value was used to conclude the differences in growth pattern between the groups. Error for mean of means is propagated by weighted pooled variance.

\section{Results}

\section{Ehmt2 (G9a) is dispensable in skeletal muscle development}

To examine the role of Ehmt2 in myogenesis in vivo, we first established a transgenic mouse model in which the Ehmt2 gene was conditionally knocked out in the skeletal muscle lineage. Mice harboring loxP-flanked Ehmt2 alleles (Ehmt $2^{\text {floxed }}$ ) were crossed to mice with a Cre recombinase gene knocked in to the Myod locus $\left(\right.$ Myod $\left.^{\text {Cre }}\right)$ [34]. To verify the efficiency of the conditional knockout, we performed an exon-specific allele-counting assay using digital droplet PCR to measure functional allele frequency [19] (see the "Methods" section for details). In FACS-purified satellite cells from control mice $\left(\right.$ Myod $^{w t / w t}$ Ehmt $2^{\text {floxed/floxed }}$ ), the Ehmt2 functional allele frequency was $100 \%$; whereas in the knockout mice (Myod $^{w t / C r e}$ Ehmt $\left.2^{\text {floxed/floxed }}\right)$, the functional allele frequency was reduced to $2.9-7.9 \%$ (95\% CI) (Fig. 1a). These genomic results were consistent with immunostaining quantification of EHMT2 protein in satellite cells. In wildtype mice, EHMT2 was robustly expressed in activated satellite cells whereas no detectable staining was present in the knockout mice (Fig. 1c). Furthermore, western blot analysis of whole skeletal muscle lysates from the conditional knockout mice showed reduction of H3K9me2 levels compared to those of the wildtype (Fig. 1b), congruent with previous reports that H3K9me2 is diminished in Ehmt $2^{\text {null/null }}$ models [14].

Knockout and control group progenies from $\mathrm{Myod}^{\text {Cre }}$ and Ehmt $2^{\text {floxed }}$ breeding were born at expected Mendelian frequencies (Fig. 1d), and neonatal weights were similar between both groups (Fig. 1e). Growth patterns of knockout and control group progenies were charted by body weight, which showed no statistically significant differences (Fig. 1f). Mature skeletal muscles in these mice are of similar size (Fig. 1g) and showed no difference upon histological examination, which included a comparison of

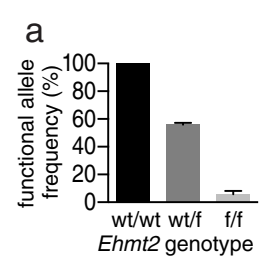

b

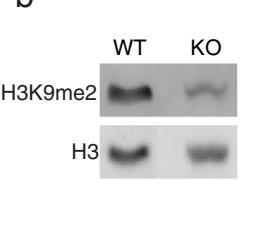

d

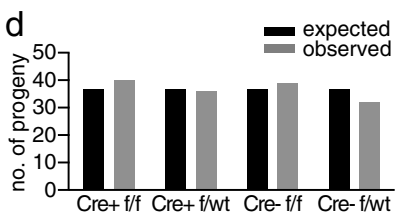

f

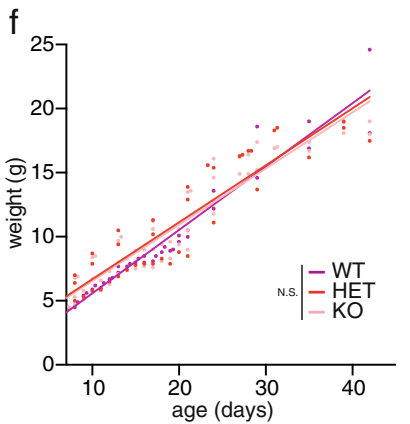

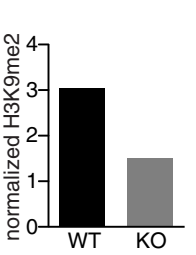

e
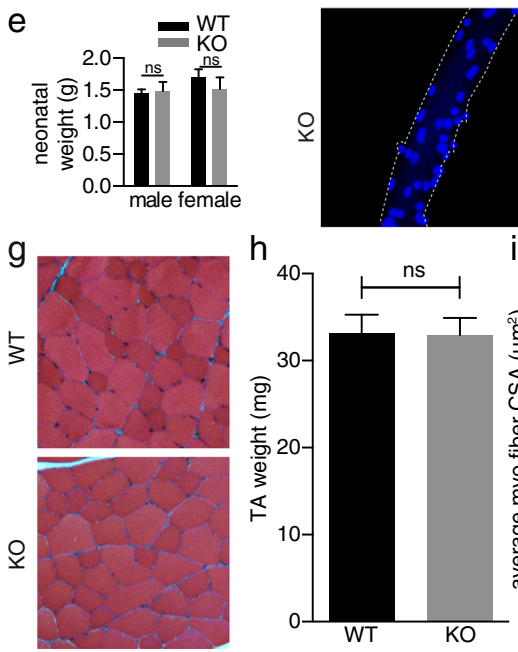
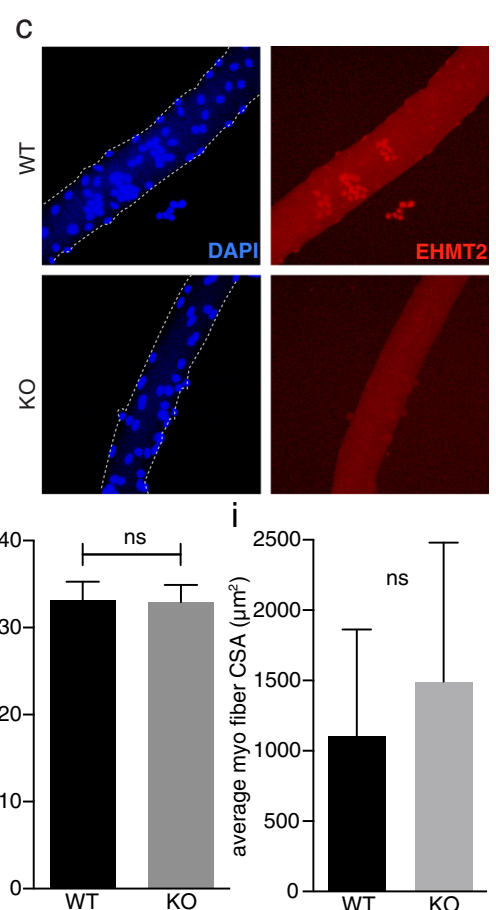

Fig. 1 Ehmt2 is dispensable for normal muscle development. a Ehmt2 deletion efficiency as measured by functional allele frequency in FACS-purified satellite cells. Analysis by gDNA allele counting using ddPCR, $n \geq 3$. b Relative abundance of H3K9me2 in whole skeletal muscle tissue lysate of wildtype and knockout mice, normalized to histone H3. c Immunofluorescence detection of EHMT2 on myofiber. $\mathbf{d}$ Number of live births from $n \geq 3$ mating pairs of Myod ${ }^{C r e}$ Ehmt $2^{\text {floxed/floxed }}$ mice. e, $\mathbf{f}$ Neonatal weight and growth curve of wildtype and knockout mice at $D_{0}-D_{1}$. $\mathbf{g}$ Masson's trichrome stain of histological sections of the tibialis anterior muscle of adult mice. $\mathbf{h}$ Whole muscle weight. $\mathbf{i}$ Myofiber size measurement by cross-sectional area 
myofiber size by measuring the cross-sectional area of myofibers (Fig. 1h, i). These findings indicate that Ehmt2 in the skeletal muscle lineage is dispensable in embryonic and fetal development.

\section{Ehmt2 (G9a) knockout satellite cells have normal} proliferation kinetics and differentiation capacity in vitro As previous reports have suggested that Ehmt2 is an important regulator of $\mathrm{C} 2 \mathrm{C} 12$ myogenesis, we next assessed whether it plays a similar role in satellite cell and primary myoblast cultures ex vivo. To analyze satellite cell proliferation, we performed a 4-h 5-ethynyl-2'-deoxyuridine (EdU) pulse on myofiber-associated satellite cells from the wildtype and conditional knockout mice after $72 \mathrm{~h}$ in culture. No differences in EdU incorporation were detected between the control and knockout groups (Fig. 2a, e). Similarly, quantification of immunofluorescent staining showed similar numbers of PAX7+ (Fig. 2c) and MYOD+ (Fig. 2b) satellite cells after $72 \mathrm{~h}$ in culture. These results indicate that satellite cells lacking Ehmt2 show comparable rates of proliferation and myogenic activation to wild-type cells ex vivo.

Next, we assessed the requirement of Ehmt2 for satellite cells to undergo myogenic differentiation. Satellite cell-derived myofibers from wildtype (WT) and knockout $(\mathrm{KO})$ mice were expanded to confluence, induced to differentiate, and then analyzed by immunostaining of MYOG and myosin heavy chain after 4, 24, and $48 \mathrm{~h}$. Under differentiating conditions, MYOG-expressing cells increased, but no significant differences in the percentages of cells expressing MYOG were observed between control and $\mathrm{KO}$ myoblasts (Fig. 2e). Similarly, we found no significant differences in myogenic fusion index (ratio of fused nuclei found in myosin-expressing cells to total nuclei) following $48 \mathrm{~h}$ of differentiation (Fig. 2f), providing further support that deletion of Ehmt2 does not have significant effects on the progress or timing of myogenic differentiation in primary myoblasts.

Together, these data provide little evidence that Ehmt2 plays a major role in the regulation of satellite cell proliferation or myogenic differentiation in vitro.

\section{Skeletal muscle- and satellite cell-specific deletion of Ehmt2 (G9a) has little effect on muscle regeneration in vivo}

Before evaluating the requirement of Ehmt2 in the response of skeletal muscle to acute injury in vivo, we first analyzed the expression of the gene in our injury model in WT mice, which involved an intramuscular injection of notexin (a snake venom toxin) in the TA muscle. During the ensuing regenerative process, we performed transcriptome sequencing of WT primary myoblasts at
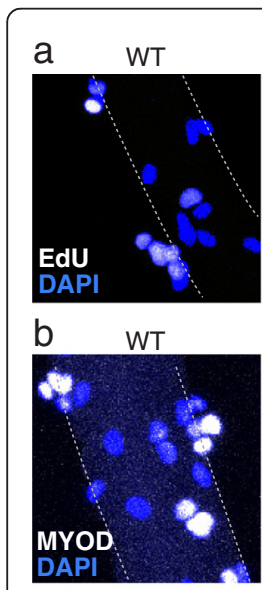

C
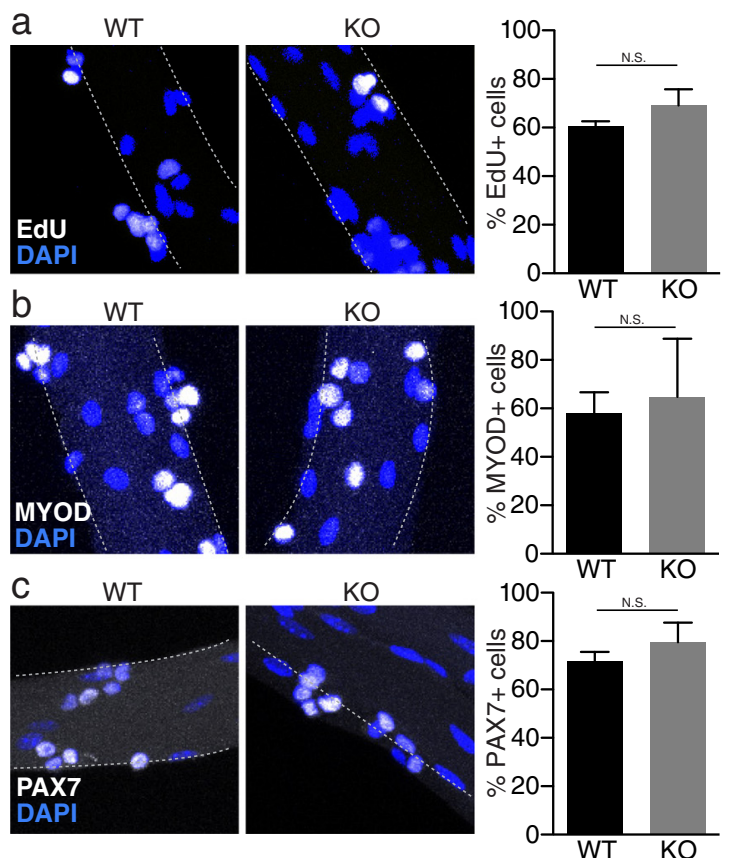
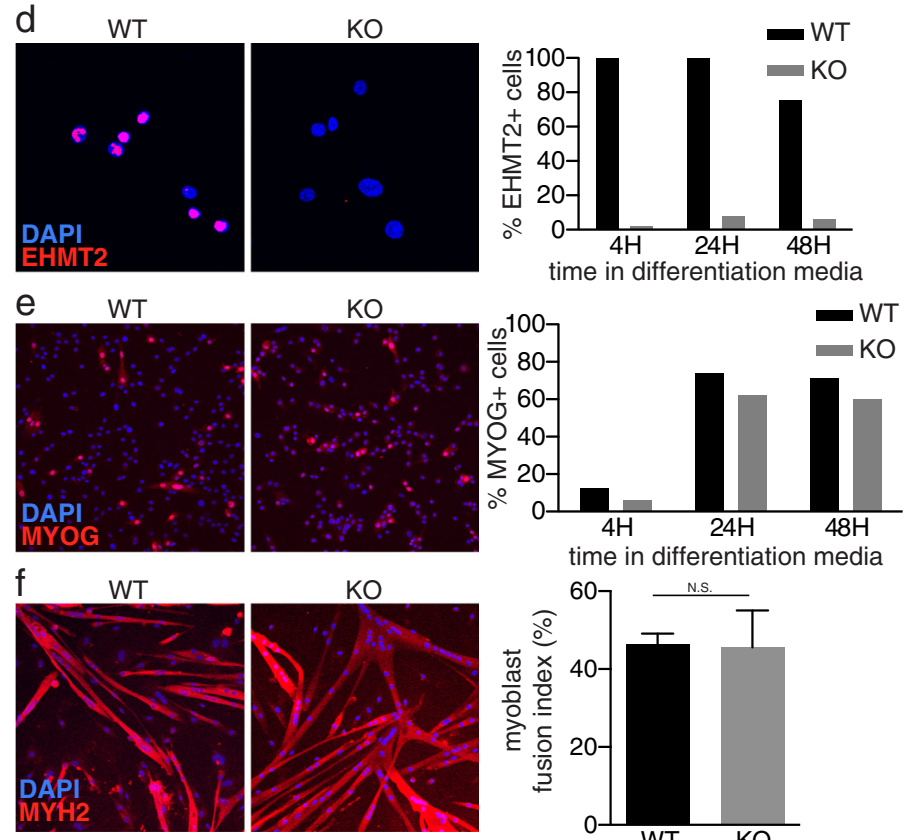

KO
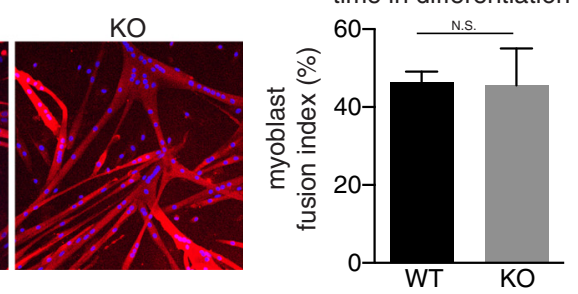

Fig. 2 Ehmt2 knockout satellite cells proliferate and differentiate normally. Myofibers were isolated from WT and Myod-Cre Ehmt2 KO mice and cultured under growth conditions for $72 \mathrm{~h}$ and satellite cells visualized by confocal microscopy. a Detection of proliferating cells after $4 \mathrm{~h}$ of EdU treatment. $\mathbf{b}, \mathbf{c}$ Immunofluorescence detection of MYOD and PAX7. Myoblasts were seeded at equal densities and induced with differentiation media for 4, 24, and 48 h. d, e Immunofluorescence detection of EHMT2 and MYOG, respectively. $\mathbf{f}$ Immunofluorescence detection of myosin heavy chain in myotubes, myoblast fusion index calculated as \% nuclei inside myosin-expressing myotubes 
different timepoints; in our results, neither Ehmt2 nor Ehmt1 showed any dynamic changes in expression during the process, in contrast to key myogenic regulators (Additional file 1: Figure S2).

Then, we evaluated the aforementioned Myod ${ }^{C r e}$

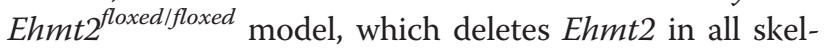
etal muscles and satellite cells during their development [39]. Following injury, we quantified the cross-sectional area of centrally nucleated myofibers as a measure of regeneration [40]. Despite a trend toward an increased number of the largest fibers in KO samples, no statistically significant difference was found between the control and $\mathrm{KO}$ mice in the distribution of myofiber size at 7,14 , or 21 days post injury, indicating comparable regenerative capacities (Fig. 3b, Additional file 1: Figure S3).

Myod-driven CRE leads to target deletion early in development and could therefore trigger compensatory effects that mask the regulatory role of Ehmt2 in adult regenerative myogenesis. To mitigate this risk, we performed a satellite cell-specific, inducible Ehmt $2 \mathrm{KO}$ using a strain carrying the tamoxifen-activated CreERT2 recombinase knocked in to the $\operatorname{Pax} 7$ locus [35]. This allowed us to confine the gene deletion within adult satellite cells and to use a Rosa $26^{Y F P}$ reporter to monitor the efficiency of induction. One week after the end of tamoxifen treatment, we found that the YFP reporter was activated in $70 \%$ of cells in the satellite cell population (Additional file 1: Figure S4). Since this was not a definitive measure of gene deletion, we further performed Ehmt2 allele count and found that the functional allele frequency had been reduced to $27.2 \pm 4.2 \%$ (SEM) in the satellite cell population purified by FACS.

Acute muscle injury by notexin was performed at 7 days after the final CreERT2 induction on mice harboring Pax $7^{\text {CreERT2 }}$ and Ehmt $2^{\text {floxed/null }}$ alleles. At 7, 14, and 21 days after the injury, no significant differences in myofiber size distribution were observed between the control and $\mathrm{KO}$ groups (Fig. 3f, Additional file 1: Figure S5, data not shown), suggesting that lack of Ehmt2 in adult satellite cells does not significantly affect repair and regeneration in vivo.

Together, our data provide little evidence for a role of Ehmt2 in regulating skeletal muscle development, homeostasis, or regeneration in vivo.

\section{Discussion}

In this study, we provide a comprehensive assessment of the biological consequences of G9a deletion in skeletal muscle progenitors. Our data strongly suggests that Ehmt2 is dispensable for both developmental and regenerative myogenesis in vivo and is not required for normal satellite cell proliferation and myogenic differentiation in vitro.
Although germ line deletion of Ehmt2 is embryonically lethal, conditional $\mathrm{KO}$ models suggest that Ehmt2 plays distinct roles in different tissues. Studies have shown an involvement of Emht 2 in the regulation of embryogenesis [14, 28, 41], cardiac morphogenesis [16], lymphopoiesis [17, 18], myelopoiesis [19], germ cell development [15], brain and cognitive development [21, 22], and drug addiction [20], confirming Ehmt2 is capable of controlling a diverse range of biological processes. In the case of myogenesis, we showed that conditional loss of Ehmt2 in vivo does not induce any significant developmental impact or any significant alterations to the regenerative capacity of myogenic progenitors in response to skeletal muscle injury. Deletion of Ehmt2 in primary myoblasts also fails to induce any significant alterations in proliferative kinetics or differentiation capacity in vitro, suggesting little role for Ehmt2 in regulating myogenesis.

These results are surprising given the previous reports suggesting an important role for Ehmt2 in negatively regulating myogenic differentiation of $\mathrm{C} 2 \mathrm{C} 12$ cells, an immortalized myogenic line. In particular, it was demonstrated that siRNA knockdown of Ehmt2 led to enhanced and/or premature differentiation of $\mathrm{C} 2 \mathrm{C} 12$ myoblasts [31]. It was further reported that Ehmt2 is an integral component of the mechanism with which Bhlhe41/Sharp1 regulates in myogenesis, in that it is recruited by SUMOylated BHLHE41/SHARP1 [33], methylates MYOD at lysine 104 [31], and leads to repressive H3K9me2 modifications on Myod targets [32]. These results were not consistent with observations in the current study when examining the differentiation capacity of primary myoblasts lacking Ehmt2, which showed no premature differentiation, no alterations in myogenic fusion, and normal proliferation. This discrepancy in findings could stem from the fundamental differences between the biological models being analyzed; unlike $\mathrm{C} 2 \mathrm{C} 12$ cells, which were derived from a different strain of mouse ( $\mathrm{C} 3 \mathrm{H})$ [42], immortalized [43], and have a much shorter doubling time [44], the primary myoblasts in our study were not serially passaged and were analyzed in their myofiber niche during proliferation and on Matrigel during differentiation, in addition to in vivo analyses. These differences may be particularly relevant in the case of Ehmt2, as we have recently reported, in another tissue system, that its absence has drastically different effects on transformed compared to natural hematopoietic cells [19].

To date, no genome-wide analysis of the Ehmt2-mediated H3K9me2 in myogenic cells exists. Dynamic changes in H3K9me2 have been reported at specific gene bodies and regulatory regions $[45,46]$, suggesting that modulating this epigenetic mark may affect gene transcription. However, in our experiments, lack of Ehmt2 led to a dramatic drop in the global levels of 


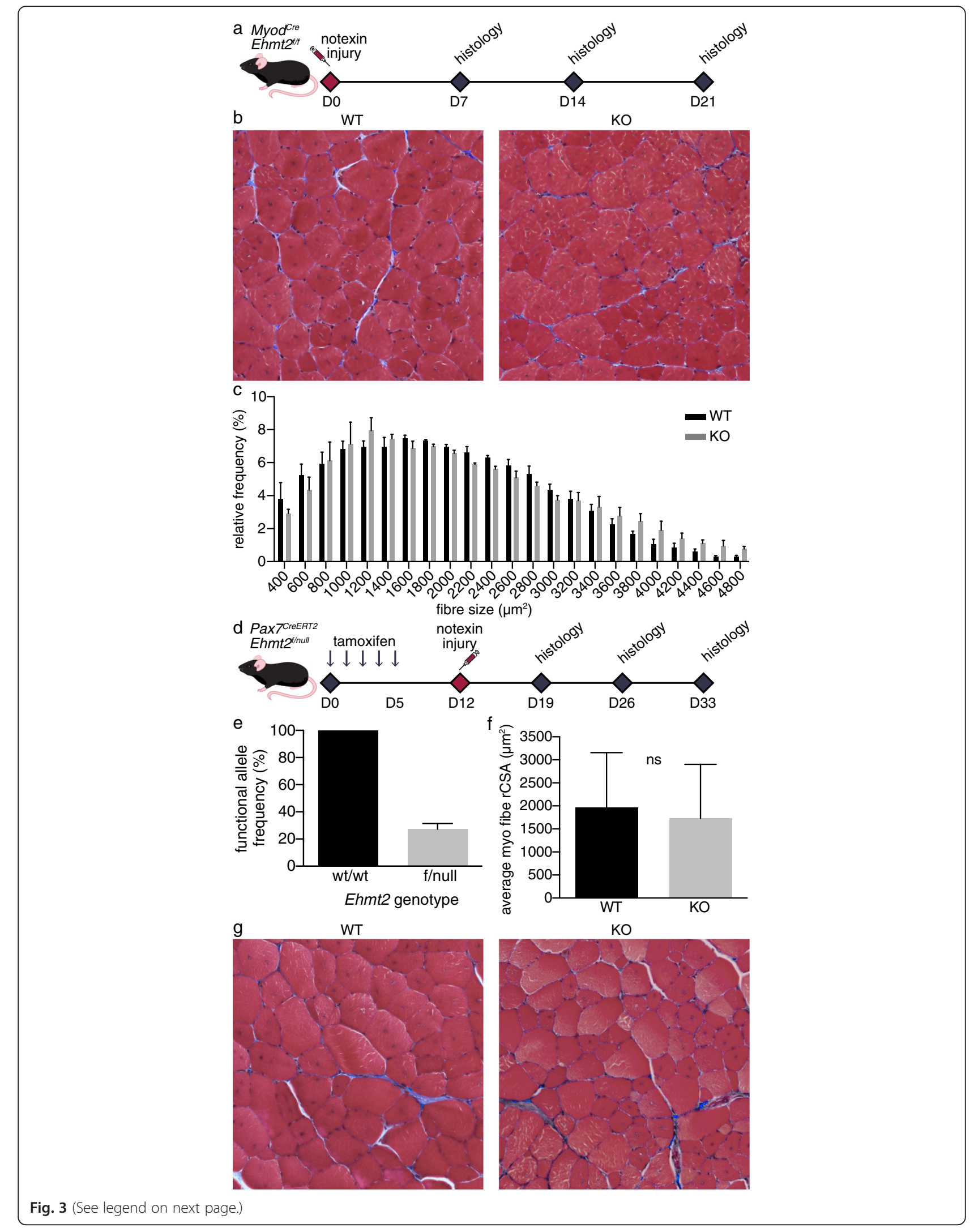


(See figure on previous page.)

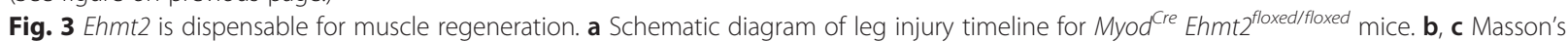
trichrome stain of histological sections of the tibialis anterior muscle of adult Myod ${ }^{\text {cre }}$ Ehmt ${ }^{\text {floxed/floxed }}$ mice at 21 days after injury and myofiber size measurement by cross-sectional area, respectively. $\mathbf{d}$ Schematic diagram of leg injury timeline for Pax CreERT2 Ehmt floxed/null mice. e Ehmt2 deletion efficiency by Pax $7^{\text {CreERT2 }}$ as measured by functional allele frequency in FACS-purified satellite cells. Analysis by gDNA allele counting using $\mathrm{ddPCR}, n \geq 3$. $\mathbf{f}, \mathbf{g}$ Myofiber size measurement by cross-sectional area and Masson's trichrome stain of histological sections of the tibialis anterior

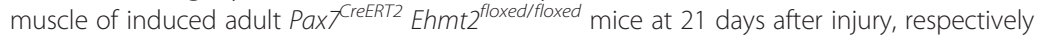

detectable H3K9me2 in the absence of any effects on skeletal muscle development. This indicates that Ehmt2 activity is mostly non-redundant and questions the importance of Ehmt2-mediated histone modifications in myogenesis in particular and in the control of differentiation in general.

What remains unclear is the status of EHMT2mediated methylation of MYOD at lysine 104 [31] in the in vivo model. Ling et al. reported the identification of this residue by mass spectrometry of peptides resulting from the digestion of MYOD with trypsin [31]. However, the reported MYOD peptides, ACKACKRKTT and its methylated forms, do not appear to be obtainable by trypsin digestion alone or by any commonly used digestion method. The reported MYOD peptide is also not found in the tandem mass spectrometry proteomics repository PeptideAtlas (https://db.systemsbiology.net/ sbeams/cgi/shortURL?key=1mk36ybs). More intriguingly, the proposed mechanism [31] was based on liquid chromatography-mass spectrometry (LC-MS) results, showing that the different methylation states of the MYOD peptide are separated by only $1 \mathrm{~m} / \mathrm{z}$ unit each. Methylation adds $14 \mathrm{Da}$ to the peptide mass; thus, each peptide would have to carry 14 charges on 10 residues. The LC-MS results could not possibly correspond to methylation of the reported MYOD peptide. The uncertainty of MYOD methylation, together with the dispensability of Ehmt2-mediated H3K9me2 in vivo, casts doubts on the proposed role of Ehmt2 in Bhlhe41/Sharp1-mediated regulation of myogenesis [32]. Nevertheless, SUMOylated Bhlhe41/Sharp1 [33] may still regulate Myod and downstream targets through an alternative mechanism.

Although we have shown here that the loss of EHMT2's histone methylation function in our model was not compensated, the possibility exists that its potential interaction with myogenic regulators could be compensated by another gene, such as its close homologue Ehmt1 (GLP). These two genes are highly similar in structure, as their protein products contain highly similar catalytic domains for lysine methylation (SET domain) and a set of ankyrin repeats for protein-protein interaction. These two enzymes are known to form heterodimers [14] but also play unique roles depending on the cell type and developmental stage [47]. Using domain-specific mutations, the EHMT1 but not the EHMT2 ankyrin repeats were found to be required for mouse viability [48], suggesting that this domain function in EHMT2 could be compensated by EHMT1. On the other hand, the SET domain in EHMT1 is dispensable for mouse viability [16], suggesting EHMT2 could compensate for EHMT1's methyltransferase activity. In our Ehmt2 KO cells, even though the histone methyltransferase activity, a function shared by both proteins, is not compensated by EHMT1, its ankyrin repeatdependent protein-protein interactions may be compensated. Both proteins have been reported to methylate a number of non-histone targets beyond MYOD [49, 50], and it is unknown if EHMT1 could replace EHMT2 in binding and methylating these targets. Interestingly, such compensation by Ehmt1 was not observed in $\mathrm{C} 2 \mathrm{C} 12$ studies [31], and in our transcriptome data, neither of the genes showed any dynamic changes during regeneration. Nevertheless, to fully address this concern, a conditional double $\mathrm{KO}$ of Ehmt2 and Ehmt1 in myogenesis would be required.

\section{Conclusions}

In this study, we analyzed tissue-specific $\mathrm{KO}$ models of Ehmt2 both during development and in adult satellite cells, and unlike as previously reported, we found no evidence for a significant role of this methyltransferase in the development or regeneration of skeletal muscle. The drop in H3K9me2 levels observed in cells lacking Ehmt2 strongly suggests that this histone modification is dispensable for the regulation of myogenesis. Primary cultures revealed that Ehmt2 does not significantly alter the proliferation and differentiation processes of satellite cells. Thus, the proposed regulatory role of Ehmt 2 in myogenesis cannot be validated in vivo.

\section{Additional file}

Additional file 1: Main figures are referred to as Fig. \# in the article. Figure legends are supplied on the next page. Supplementary figures with figure legends are referred to as Supplementary Fig. \# in the article.

\section{Abbreviations}

APC: allophycocyanin; bHLH: basic helix-loop-helix; Bhlhe41: basic helix-loophelix family member e41, aka Sharp1; CCAC: Canadian Council on Animal Care; ChIP: chromatin immunoprecipitation; Cl: confidence interval; Cre: Causes Recombination; ddPCR: digital droplet polymerase chain reaction; DMEM: Dulbecco's modified Eagle's medium; DNMT: DNA methyltransferase; DSHB: Developmental Studies Hybridoma Bank; EDL: extensor digitorum longus; EdU: 5-ethynyl-2'-deoxyuridine; Ehmt1: euchromatic histone-lysine N-methyltransferase 1, aka GLP (G9a-like 
protein); Ehmt2: euchromatic histone-lysine $\mathrm{N}$-methyltransferase 2, aka G9a; FACS: fluorescence-activated cell sorting; FAM: fluorescein amidite; FITC: fluorescein isothiocyanate; H2B: histone 2B; H3K27ac: acetylation of lysine 27 on histone $\mathrm{H} 3$ protein subunit; $\mathrm{H} 3 \mathrm{~K} 4 \mathrm{me}$ 1: unimethylation of lysine 4 on histone $\mathrm{H3}$ protein subunit; H3K9me2: trimethylation of lysine 9 on histone $\mathrm{H} 3$ protein subunit; $\mathrm{HP} 1$ : heterochromatin protein 1; KO: knockout; LC-MS: liquid chromatography-mass spectrometry; LY6A/E: Iymphocyte antigen 6A/E, aka SCA-1 (stem cell antigen 1); MRF: myogenic regulatory factor; Mrf4: myogenic regulatory factor 4, aka myogenic factor 6 (herculin); Myf5: myogenic factor 5; Myog: myogenin; Pax7: paired box 7;

PE: phycoerythrin; PECAM: platelet endothelial cell adhesion molecule, aka CD31 (cluster of differentiation 31); PE-Cy7: phycoerythrin-cyanine 7 tandem complex; PTPRC: protein tyrosine phosphatase receptor type C, aka CD45 (cluster of differentiation 45); SEM: standard error of mean; SET: Su (var)3-9 and enhancer of zeste; Shh: sonic hedgehog; SUMO: small ubiquitin-like modifier; TA: tibialis anterior; VCAM: vascular cell adhesion molecule, aka CD106 (cluster of differentiation 106); WT: wildtype.

\section{Acknowledgements}

We thank all the lab members and alumni for the technical and scientific support, especially Ms Lin Yi, Mr Chihkai Chang, Ms Vittoria Canale, Ms Claudia Hopkins, Dr Bernhard Lehnertz, Dr Dario Lemos, Dr Pretheeban Thavaneetharajah, Dr Anuradha Natarajan, Dr Farshad Babaei, Ms Christine Eisner, Dr Coral-Ann Lewis, Dr Marcela Low, Ms Joey Nguyen, Dr Hesham Soliman, Ms Gloria Loi, Mr Alan Wong, Mr Ryan Cheng, Mr Alvin Tsuei, and especially Dr Elena Groppa for the transcriptome-sequencing database of skeletal muscle regeneration. The Animal Unit staff at The Biomedical Research Centre were instrumental in supporting the in vivo research, especially Ms Helen Merkens, Ms Krista Ranta, Mr Wei Yuan, and Mr Jerry Chen. We also owe tremendous help from Mr Andy Johnson and Mr Justin Wong of UBC Flow Cytometry, Ms Ingrid Barta of the UBC Histology Lab, and Mr John Schipilow and Dr Nancy Ford of the Centre for High-Throughput Phenogenomics.

\section{Funding}

This research is supported by the Canadian Institutes of Health Research grant number MOP-97856.

\section{Availability of supporting data}

Not applicable.

\section{Authors' contributions}

$\mathrm{RHZ}$ contributed to the conception of the strategies, project management, design of the experiments, carrying out the in vivo and in vitro experiments, data analysis, statistical analysis, writing all the sections of the article, and figure designs. RNJ contributed to the design of the in vitro experiments, carrying out the myofiber culture experiments, writing the "Methods" section for cell culture and immunocytochemistry, and editing of the article. DYL contributed to carrying out the in vivo experiments, collecting the data from microscopy, and data analysis. JK contributed to writing the discussion. FMR contributed to the conception of the strategies, supervision of the project, design of the experiments, and writing the background, results, discussion, abstract, and all the correspondences. All authors read and approved the final manuscript.

\section{Competing interests}

The authors declare that they have no competing interests.

\section{Consent for publication}

$$
\text { Not applicable. }
$$

\section{Ethics approval and consent to participate}

We obtained ethical approval from the UBC Animal Care and Use Program.

\section{Received: 8 April 2016 Accepted: 17 May 2016 \\ Published online: 27 May 2016}

\section{References}

1. Parker MH, Seale P, Rudnicki MA. Looking back to the embryo: defining transcriptional networks in adult myogenesis. Nat Rev Genet. 2003;4(7):497-507.
2. Borycki AG, Brunk B, Tajbakhsh S, Buckingham M, Chiang C, Emerson Jr CP. Sonic hedgehog controls epaxial muscle determination through Myf5 activation. Development. 1999;126(18):4053-63.

3. Gustafsson MK, Pan H, Pinney DF, Liu Y, Lewandowski A, Epstein DJ, et al. Myf5 is a direct target of long-range Shh signaling and Gli regulation for muscle specification. Genes Dev. 2002;16(1):114-26.

4. Tajbakhsh S, Borello U, Vivarelli E, Kelly R, Papkoff J, Duprez D, et al. Differential activation of Myf5 and MyoD by different Wnts in explants of mouse paraxial mesoderm and the later activation of myogenesis in the absence of Myf5. Development. 1998;125(21):4155-62.

5. Sartorelli V, Juan AH. Sculpting chromatin beyond the double helix: epigenetic control of skeletal myogenesis. In: Pavlath GK, editor. Myogenesis. United States: Elsevier Inc; 2011. p. 57-83.

6. Sartorelli V, Puri PL, Hamamori Y, Ogryzko V, Chung G, Nakatani Y, et al. Acetylation of MyoD directed by PCAF is necessary for the execution of the muscle program. Mol Cell. 1999;4(5):725-34.

7. Polesskaya A, Duquet A, Naquibneva I, Weise C, Vervisch A, Bengal E, et al. CREB-binding protein/p300 activates MyoD by acetylation. J Biol Chem. 2000;275(44):34359-64.

8. Berkes CA, Tapscott SJ. MyoD and the transcriptional control of myogenesis. Semin Cell Dev Biol. 2005:16(4-5):585-95.

9. Asp P, Blum R, Vethantham V, Parisi F, Micsinai M, Cheng J, et al. Genomewide remodeling of the epigenetic landscape during myogenic differentiation. Proc Natl Acad Sci U S A. 2011;108(22):E149-58.

10. Vethantham V, Yang Y, Bowman C, Asp P, Lee JH, Skalnik DG, et al. Dynamic loss of $\mathrm{H} 2 \mathrm{~B}$ ubiquitylation without corresponding changes in H3K4 trimethylation during myogenic differentiation. Mol Cell Biol. 2012;32(6):1044-55.

11. Blum R, Vethantham V, Bowman C, Rudnicki M, Dynlacht BD. Genome-wide identification of enhancers in skeletal muscle: the role of MyoD1. Genes Dev. 2012;26(24):2763-79.

12. Blum R, Dynlacht BD. The role of MyoD1 and histone modifications in the activation of muscle enhancers. Epigenetics. 2013;8(8):778-84.

13. Rea S, Eisenhaber F, O'Carroll D, Strahl BD, Sun ZW, Schmid M, et al. Regulation of chromatin structure by site-specific histone $\mathrm{H3}$ methyltransferases. Nature. 2000;406(6796):593-9.

14. Tachibana M, Sugimoto K, Nozaki M, Ueda J, Ohta T, Ohki M, et al. G9a histone methyltransferase plays a dominant role in euchromatic histone $\mathrm{H} 3$ lysine 9 methylation and is essential for early embryogenesis. Genes Dev. 2002;16(14):1779-91.

15. Tachibana M, Nozaki M, Takeda N, Shinkai Y. Functional dynamics of H3K9 methylation during meiotic prophase progression. EMBO J. 2007;26(14): 3346-59.

16. Inagawa M, Nakajima K, Makino T, Ogawa S, Kojima M, Ito S, et al. Histone H3 lysine 9 methyltransferases, G9a and GLP are essential for cardiac morphogenesis. Mech Dev. 2013;130(11-12):519-31.

17. Lehnertz B, Northrop JP, Antignano F, Burrows K, Hadidi S, Mullaly SC, et al. Activating and inhibitory functions for the histone lysine methyltransferase G9a in T helper cell differentiation and function. J Exp Med. 2010;207(5): 915-22.

18. Thomas LR, Miyashita H, Cobb RM, Pierce S, Tachibana M, Hobeika E, et al. Functional analysis of histone methyltransferase g9a in B and T lymphocytes. J Immunol. 2008;181(1):485-93.

19. Lehnertz B, Pabst C, Su L, Miller M, Liu F, Yi L, et al. The methyltransferase G9a regulates HoxA9-dependent transcription in AML. Genes Dev. 2014;28(4):317-27.

20. Maze I, Covington III HE, Dietz DM, LaPlant Q, Renthal W, Russo SJ, et al. Essential role of the histone methyltransferase G9a in cocaine-induced plasticity. Science. 2010;327(5962):213-6.

21. Schaefer A, Sampath SC, Intrator A, Min A, Gertler TS, Surmeier DJ, et al. Control of cognition and adaptive behavior by the GLP/G9a epigenetic suppressor complex. Neuron. 2009;64(5):678-91.

22. Kramer JM, Kochinke K, Oortveld MA, Marks H, Kramer D, De Jong EK, et al. Epigenetic regulation of learning and memory by Drosophila EHMT/G9a. PLoS Biol. 2011;9(1), e1000569.

23. Lee DY, Northrop JP, Kuo MH, Stallcup MR. Histone H3 lysine 9 methyltransferase G9a is a transcriptional coactivator for nuclear receptors. J Biol Chem. 2006; 281(13):8476-85.

24. Sampath SC, Marazzi I, Yap KL, Sampath SC, Krutchinsky AN, Mecklenbrauker l, et al. Methylation of a histone mimic within the histone methyltransferase G9a regulates protein complex assembly. Mol Cell. 2007;27(4):596-608. 
25. Huang J, Dorsey J, Chuikov S, Perez-Burgos L, Zhang X, Jenuwein T, et al. G9a and Glp methylate lysine 373 in the tumor suppressor p53. J Biol Chem. 2010;285(13):9636-41.

26. Huang J, Berger SL. The emerging field of dynamic lysine methylation of non-histone proteins. Curr Opin Genet Dev. 2008;18(2):152-8.

27. Mosavi LK, Cammett TJ, Desrosiers DC, Peng ZY. The ankyrin repeat as molecular architecture for protein recognition. Protein Sci. 2004;13(6):1435-48

28. Dong KB, Maksakova IA, Mohn F, Leung D, Appanah R, Lee S, et al. DNA methylation in ES cells requires the lysine methyltransferase G9a but not its catalytic activity. EMBO J. 2008;27(20):2691-701.

29. Epsztejn-Litman S, Feldman N, Abu-Remaileh M, Shufaro Y, Gerson A, Ueda J, et al. De novo DNA methylation promoted by G9a prevents reprogramming of embryonically silenced genes. Nat Struct Mol Biol. 2008;15(11):1176-83.

30. Purcell DJ, Jeong KW, Bittencourt D, Gerke DS, Stallcup MR. A distinct mechanism for coactivator versus corepressor function by histone methyltransferase G9a in transcriptional regulation. J Biol Chem. 2011;286(49):41963-71.

31. Ling BM, Bharathy N, Chung TK, Kok WK, Li S, Tan YH, et al. Lysine methyltransferase G9a methylates the transcription factor MyoD and regulates skeletal muscle differentiation. Proc Natl Acad Sci U S A. 2012;109(3):841-6.

32. Ling BM, Gopinadhan S, Kok WK, Shankar SR, Gopal P, Bharathy N, et al. G9a mediates Sharp-1-dependent inhibition of skeletal muscle differentiation. Mol Biol Cell. 2012;23(24):4778-85.

33. Wang Y, Shankar SR, Kher D, Ling BM, Taneja R. Sumoylation of the basic helix-loop-helix transcription factor sharp-1 regulates recruitment of the histone methyltransferase G9a and function in myogenesis. J Biol Chem. 2013;288(24):17654-62.

34. Kanisicak O, Mendez JJ, Yamamoto S, Yamamoto M, Goldhamer DJ. Progenitors of skeletal muscle satellite cells express the muscle determination gene. MyoD Dev Biol. 2009;332(1):131-41.

35. Lepper C, Conway SJ, Fan CM. Adult satellite cells and embryonic muscle progenitors have distinct genetic requirements. Nature. 2009;460(7255):627-31.

36. Srinivas $S$, Watanabe $T$, Lin CS, William CM, Tanabe $Y$, Jessell TM, et al. Cre reporter strains produced by targeted insertion of EYFP and ECFP into the ROSA26 locus. BMC Dev Biol. 2001;1:4.

37. Joe AW, Yi L, Natarajan A, Le Grand F, So L, Wang J, et al. Muscle injury activates resident fibro/adipogenic progenitors that facilitate myogenesis. Nat Cell Biol. 2010;12(2):153-63.

38. Collins CA, Zammit PS. Isolation and grafting of single muscle fibres. Methods Mol Biol. 2009:482:319-30.

39. Wood WM, Etemad S, Yamamoto M, Goldhamer DJ. MyoD-expressing progenitors are essential for skeletal myogenesis and satellite cell development. Dev Biol. 2013;384(1):114-27.

40. Murphy MM, Lawson JA, Mathew SJ, Hutcheson DA, Kardon G. Satellite cells, connective tissue fibroblasts and their interactions are crucial for muscle regeneration. Development. 2011;138(17):3625-37.

41. Yamamizu K, Fujihara M, Tachibana M, Katayama S, Takahashi A, Hara E, et al. Protein kinase A determines timing of early differentiation through epigenetic regulation with G9a. Cell Stem Cell. 2012;10(6):759-70.

42. Yaffe D, Saxel O. Serial passaging and differentiation of myogenic cells isolated from dystrophic mouse muscle. Nature. 1977;270(5639):725-7.

43. Blau HM, Pavlath GK, Hardeman EC, Chiu CP, Silberstein L, Webster SG, et al. Plasticity of the differentiated state. Science. 1985;230(4727):758-66.

44. Cheng CS, El-Abd Y, Bui K, Hyun YE, Hughes RH, Kraus WE, et al. Conditions that promote primary human skeletal myoblast culture and muscle differentiation in vitro. Am J Physiol Cell Physiol. 2014;306(4):C385-95.

45. Zylicz JJ, Dietmann S, Günesdogan U, Hackett JA, Cougot D, Lee C, et al. Chromatin dynamics and the role of G9a in gene regulation and enhancer silencing during early mouse development. eLife. 2015;9:4.

46. Renneville A, Van Galen P, Canver MC, McConkey M, Krill-Burger JM, Dorfman DM, et al. EHMT1 and EHMT2 inhibition induces fetal hemoglobin expression. Blood. 2015;126(16):1930-9.

47. Kramer JM. Regulation of cell differentiation and function by the euchromatin histone methyltranserfases G9a and GLP. Biochem Cell Biol. 2016;94(1):26-32.
48. Liu N, Zhang Z, Wu H, Jiang Y, Meng L, Xiong J, et al. Recognition of H3K9 methylation by GLP is required for efficient establishment of $\mathrm{H} 3 \mathrm{~K} 9$ methylation, rapid target gene repression, and mouse viability. Genes Dev. 2015;29(4):379-93.

49. Herz HM, Garruss A, Shilatifard A. SET for life: biochemical activities and biological functions of SET domain-containing proteins. Trends Biochem Sci. 2013;38(12):621-39.

50. Lanouette S, Mongeon V, Figeys D, Couture JF. The functional diversity of protein lysine methylation. Mol Syst Biol. 2014;10:724.

\section{Submit your next manuscript to BioMed Central and we will help you at every step:}

- We accept pre-submission inquiries

- Our selector tool helps you to find the most relevant journal

- We provide round the clock customer support

- Convenient online submission

- Thorough peer review

- Inclusion in PubMed and all major indexing services

- Maximum visibility for your research

Submit your manuscript at www.biomedcentral.com/submit

) Biomed Central 